\title{
BraSNAM em perspectiva: uma análise da sua trajetória até os 10 anos de existência
}

\author{
Fábio M. F. Lobato ${ }^{1,2}$, Gleyce C. de Sousa ${ }^{2}$, Antonio F. L. Jacob Jr. ${ }^{2}$ \\ ${ }^{1}$ Instituto de Engenharia e Geociências - Universidade Federal do Oeste do Pará \\ Santarém - PA - Brasil \\ ${ }^{2}$ Departamento de Engenharia da Computação - Universidade Estadual do Maranhão \\ São Luís - MA - Brasil. \\ fabio.lobato@ufopa.edu.br, antoniojunior@professor.uema.br
}

\begin{abstract}
Resumo. É inegável o aumento da pervasividade e relevância das mídias sociais em nosso cotidiano. Desde 2012, o Brazilian Workshop on Social Network Analysis and Mining (BraSNAM) representa um importante fórum para reunir pesquisadores a fim de discutir métodos de análise, tendências e fenômenos que ocorrem nas redes sociais. Neste 2021, este evento completa 10 anos, com 230 trabalhos apresentados até o momento. Além disso, possui uma comunidade composta por 527 pesquisadores e pesquisadoras de 95 diferentes instituições. À luz deste marco, esse trabalho apresenta uma análise da comunidade BraSNAM. Os resultados atestam o crescimento sustentável da comunidade, sobretudo em relação ao seu impacto técnico-científico. Os achados do trabalho podem auxiliar o comitê organizador no planejamento estratégico das próximas edições.
\end{abstract}

\section{Introdução}

A Análise de Comunidades (AC) em redes sociais tem atraído atenção crescente, devido a sua aplicabilidade nos mais diferentes segmentos, uma das tarefas realizadas é o monitoramento da evolução de comunidades dinâmicas [Chu et al. 2020, Dakiche et al. 2019]. Grande parte dos trabalhos envolvendo detecção de comunidades possui um enfoque no corte transversal, com uma análise sendo realizada em um dado momento - considerando a comunidade estática [Dakiche et al. 2019]. No entanto, a maioria das redes sociais evolui com o tempo, impulsionada pelas atividades e afiliações compartilhadas de seus membros [Kumar et al. 2010]. Uma rede dinâmica é um tipo especial de redes complexas em evolução, nas quais as mudanças ocorrem ao longo do tempo [Xu et al. 2020]. Barabási e colaboradores perceberam que as redes de colaboração científica poderiam ser um campo fértil para o estudo de redes dinâmicas [Barabási et al. 2002]. Neste trabalho, os autores mapearam bases de dados eletrônicas acerca de periódicos relevantes nas áreas de matemática e neurociências por oito anos. A análise permitiu inferir a dinâmica e os mecanismos estruturais que governam a evolução e topologia das colaborações científicas.

Além de estudos de grupos de pesquisadores e de produtividade individual, a análise de redes pode ser aplicada também ao acompanhamento de temas de programas de pesquisa. Existem várias estratégias para mapear a estrutura de conhecimento, em que a palavra-chave Rede de Coocorrência, ou, simplesmente, Rede de Palavras-Chave (RPC) se destaca pela sua objetividade, clareza e facilidade de implementação e interpretação 
[Su and Lee 2010, Radhakrishnan et al. 2017]. RPC são redes construídas a partir de correlações de palavras-chave de artigos; portanto, duas palavras que pertencem ao mesmo papel estão relacionadas entre si, formando uma borda da rede [Zhu and Guan 2013]. Dessa forma, vislumbra-se que a análise de redes sociais pode ser utilizada para analisar a colaboração de pesquisadores (co-autoria), produtividade individual (impacto) e também a evolução de assuntos (palavras-chave).

Nesta senda, considerando que o Brazilian Workshop on Social Network Analysis and Mining (BraSNAM) tem a sua décima edição neste 2021, percebemos uma oportunidade única de analisar a evolução desta comunidade. Ao iniciar o estudo, algumas perguntas norteadoras foram levantadas: i) Como a comunidade do BrasNAM evoluiu até a sua primeira década de existência? ii) Como se deu a evolução dos assuntos discutidos pela comunidade durante o evento?

Visando responder estas perguntas, conduziu-se uma análise bibliométrica/cientométrica dos trabalhos publicados no evento. Os resultados demonstram a evolução da comunidade. Algumas das análises realizadas tem o potencial de auxiliar o comitê organizador na elaboração de estratégias para a manutenção do crescimento sustentável do evento. O restante do artigo encontra-se organizado como segue. Os materiais e métodos utilizados são descritos na Seção 2. Os resultados são apresentados e discutidos na Seção 3 . Por fim, as conclusões, ameaças a validade da pesquisa e sugestão de trabalhos futuros encontram-se dispostas na Seção 4 .

\section{Materiais e Métodos}

Nesta seção apresentamos uma breve descrição do evento, as fontes de dados utilizadas e as análises conduzidas.

\subsection{Sobre o evento}

O BraSNAM ocorre anualmente como evento satélite do Congresso da Sociedade Brasileira de Computação (CSBC) desde 2012. É o evento pioneiro específico para temática relacionada à mineração e análise de redes sociais no Brasil [Digiampietri et al. 2017]. $\mathrm{O}$ evento aceita a submissão de artigos completos (até 12 páginas) e artigos curtos (até 6 páginas). Os artigos podem ser publicados em Inglês e Português.

O BraSNAM está indexado no estrato B3 do Qualis e possui H5-index igual a 5 e H5-mediana igual a 9 de acordo com as métricas do Google Acadêmicd ${ }^{1}$. Os artigos são selecionados por meio de um processo de revisão por pares do tipo duplo-cega. Em 2020 a taxa de aceitação foi de $30,61 \%$ para artigos completos e $22,22 \%$ para artigos curtos. Todos os trabalhos publicados no BraSNAM podem ser encontrados na base SBC OpenLib (SOL) ${ }^{2}$ que é uma biblioteca digital mantida pela Sociedade Brasileira de Computação (SBC). Lançada em julho de 2019, a SOL oferece acesso ao conteúdo produzido pela SBC, incluindo os artigos selecionados e apresentados nos eventos realizados ou apoiados pela SBC.

\footnotetext{
1https://scholar.google.com/citations?hl=en\&view_op=search_venues\&vq= Brazilian+Workshop+on+Social+Network+Analysistand+Mining. Acesso em: 15 de abril de 2021

2https://sol.sbc.org.br/index.php/brasnam/issue/archive Acesso em: 15 de abril de 2021
} 


\subsection{Fontes de Dados}

Neste estudo foram utilizadas quatro fontes de dados: i) a base de dados SOL, da SBC; ii) o Google Acadêmico ${ }^{3}$, iii) a API Nomes do serviço de dados do Instituto Brasileiro de Geografia e Estatística (IBGE) ${ }^{4}$; e iv) dados de redes sociais como Facebook e Twitter.

A SOL forneceu as informações básicas dos artigos, especificamente: i) título; ii) palavras-chave; iii) autores; iv) instituições; e, v) ano da publicação. Para coletar os dados, foi desenvolvido um web crawler na linguagem Python usando o framework BeautifulSoup 45. Informações adicionais foram retiradas manualmente, por exemplo, identificou-se o total de artigos submetidos por ano, taxa de aceitação de artigos e locais de realização do evento. Do Google Acadêmico se extraiu o número de citações dos artigos, os quais foram buscados pelo título. Como a plataforma não fornece API, testou-se a biblioteca scholarly $\sqrt{6}$, entretanto houve um bloqueio por parte do servidor e a coleta de dados precisou ser realizada de forma manual. Visando maior confiabilidade, os autores realizaram de forma separada e confrontaram os resultados. Em caso de inconsistência, o dado era revisado.

A API Nomes do serviço de dados do Instituto Brasileiro de Geografia e Estatística (IBGE) serviu para identificar o gênero dos autores e autoras com o intuito de se mensurar a diversidade de gênero da comunidade. A API Nomes considera a frequência de nomes registrados de acordo com o Censo. Neste ponto é importante destacar que autores estrangeiros podem não ser reconhecidos pela API (e.g. Min-Yuh Day). O mesmo ocorre com o primeiro nome suprimido, como no caso de $C$. Mateos. Por fim, considerando o uso crescente das mídias sociais para divulgação de resultados de pesquisa, o que fez surgir uma vasta gama de indicadores alternativos (Altimétricos) [Souza 2015], utilizamos em nossas análises, também dados das redes sociais Facebook e Twitter relacionados as contas oficiais do BraSNAM.

\subsection{Análises}

Em vista a riqueza de análises possíveis, restringimos algumas tarefas com enfoque nas perguntas. Dessa forma, realizamos: i) Inspeção e Pré-processamento dos dados; ii) Análise Exploratória; iii) Caracterização da Comunidade; iv) Colaborações Interinstitucionais; v) Impacto dos trabalhos; e vi) Identificação da evolução dos temas de pesquisa. Os scripts das análises foram escritos na linguagem Python. A inspeção buscou identificar eventuais falhas no processo de aquisição. Neste ponto, percebeu-se que as edições de 2017 e 2018 não dispunham de palavras-chave para todos os trabalhos publicados, e a edição de 2019 também não apresentava palavras-chave para os trabalhos curtos. Neste sentido, um trabalho de anotação manual foi realizado, baseando-se nas palavras-chave de outros estudos e na leitura do título e resumo destes estudos.

As palavras-chave, autores e instituições precisaram passar por uma etapa de préprocessamento, a fim de padronizá-los, tal como realizado em [Lobato et al. 2020]. Os passos-padrão utilizados nestes dados foram: remoção de espaços em branco duplicados ou à direita/esquerda da string, remoção de caracteres especiais e padronização para

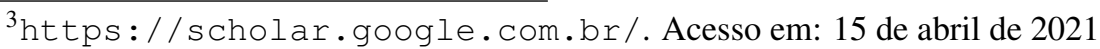

4 https://servicodados.ibge.gov.br/api/docs/nomes?versao=2. Acesso em: 15 de abril de 2021.

5 https://pypi.org/project/beautifulsoup4/. Acessado em: 09 de abril de 2021

6 https://pypi.org/project/scholarly/. Acesso em: 03 de abril de 2021
} 
caixa-baixa. Para as palavras-chave, utilizamos a medida de similaridade de strings Levenshtein, onde identificamos termos com similaridade maior que $85 \%$ e padronizamos de forma semi-automática. Por exemplo, rede de coautoria e rede de coautorias e redes de coautoria foram padronizadas para redes de coautoria. $\mathrm{O}$ processo não foi totalmente automatizado, pois em alguns casos de similaridade dentro do ponto de corte estipulado não fazia sentido o processo de padronização, por exemplo, analise de popularidade e analise de polaridade tratam de tópicos de pesquisa distintos.

Um processo semelhante foi feito para o nome dos autores. Por exemplo, $J r$. foi padronizado para Júnior. Além disso, consideramos apenas o primeiro e o último nome. O primeiro nome foi utilizado para se inferir o gênero por meio de consulta a API Nomes do IBGE. Alguns nomes não puderam ser automaticamente categorizados por não terem sido registrados no Brasil. Esses autores foram categorizados como gênero indefinido. As instituições também foram mapeadas dessa forma, as ocorrências centro federal de educacao tecnologica celso suckow da fonseca, celso suckow da fonseca federal center for technological education e cefet/rj foram transformadas em cefet-rj. Palavras-chave, autores e instituições foram pareadas por artigo, gerando uma lista de adjacências, denotando coocorrência, co-autoria e colaborações interinstitucionais, respectivamente. Utilizamos os softwares Cytoscape e Gephi para visualização dos dados e análise dos componentes das redes geradas. Para fins de replicabilidade, os dados coletados e processados encontramse disponíveis em https://github.com/fabiolobato/brasnam_2021.

\section{Resultados}

Os resultados foram organizados da seguinte forma: análise exploratória dos trabalhos publicados, caracterização da comunidade, colaborações interinstitucionais, impacto dos trabalhos e identificação dos temas mais discutidos ao longo dos anos.

\subsection{Trabalhos publicados}

De 2012 a 2020, o BraSNAM recebeu 514 submissões, tendo publicado 230 trabalhos, sendo 85 artigos curtos e 145 trabalhos completos. Isso representa uma taxa de aceitação média de $45 \%$. Cada trabalho publicado possui, em média, 3,5 autores. A distribuição temporal de submissões e trabalhos publicados pode ser vista na Figura 1 .

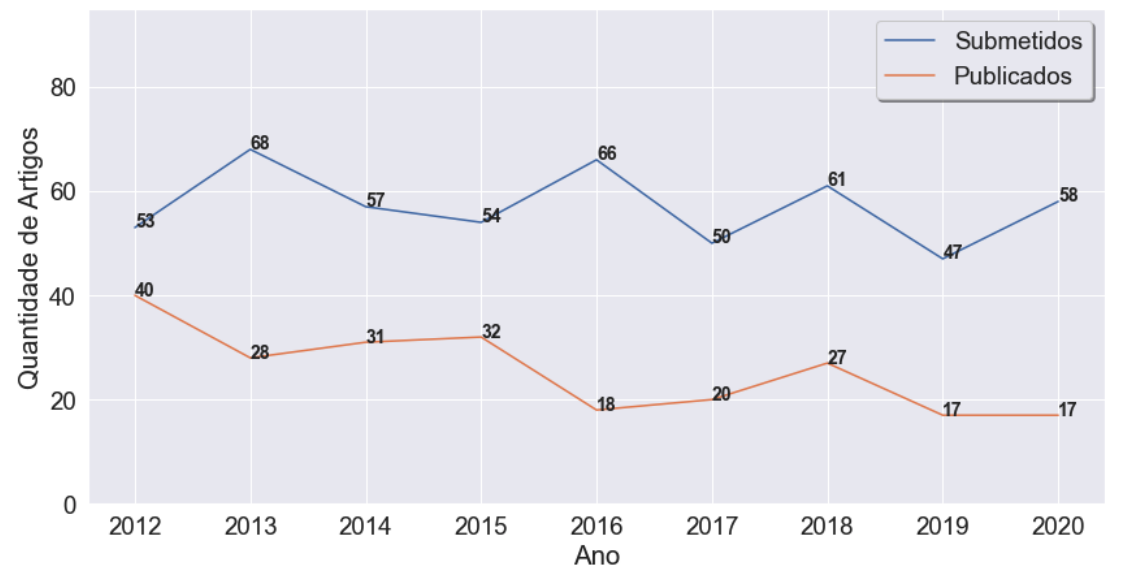

Figura 1. Distribuição da quantidade de artigos publicados ao longo dos anos. 
É interessante notar a evolução da comunidade por meio das submissões recebidas. A média e a mediana são idênticas, 57 trabalhos recebidos. No caso dos trabalhos aceitos, a média e a mediana são 25,5 e 27 trabalhos, respectivamente. Esta tendência é observável no gráfico apresentado na Figura 1, destacando a maturidade crescente da comunidade, que é corroborado pela recente inclusão no h-índice do Google Acadêmico.

\subsection{Comunidade}

Em relação a autoria, a comunidade do BraSNAM é composta por 527 pesquisadores e pesquisadoras. Em relação a distribuição por gênero, conforme mencionado na Seção anterior, utilizamos a API Nomes do IBGE e, conforme explicitado na Seção anterior, alguns nomes não puderam ser automaticamente categorizados por não terem sido registrados no Brasil. Esses autores foram categorizados como gênero indefinido e somam 20 (4\%) indivíduos. Dos 507 restantes, 374 (71\%) são do gênero masculino e 133 (25\%) do gênero feminino. De posse do gênero dos autores, cruzou-se os dados em relação aos artigos. Dos 230 trabalhos até então publicados no BraSNAM, 15 possuíam autoria exclusiva de mulheres e na 57\% (131) dos trabalhos publicados possuem ao menos uma autora. Realizamos também uma inspeção da distribuição de gêneros a cada edição, tal como apresentado na Figura 2 .

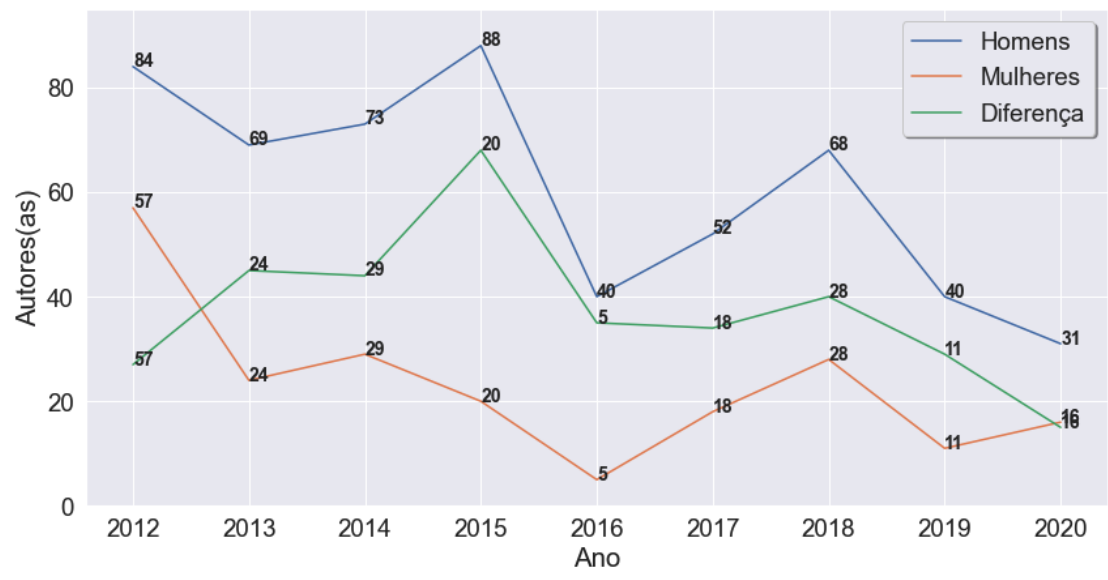

Figura 2. Distribuição de gênero por ano.

Por meio da análise dos dados dispostos na Figura 2 é possível perceber que, entre 2012 e 2015 houve um aumento na diferença de gênero, com uma tendência de mais autores do que autoras e um pico em 2015. Em 2016 houve uma queda na diferença ocorrida no ano anterior e uma tendência a estabilidade até 2018. A partir deste ano, percebe-se que a comunidade tem caminhado para uma equidade de gênero, com a maior participação de autoras.

Outra análise relacionada a comunidade foi referente a evolução da mesma ao longo dos anos. Uma pergunta norteadora feita foi: Quantos novos membros ingressam no BraSNAM a cada edição? A Figura 3 apresenta dois números. A quantidade de membros por ano (em laranja) e quantos novos membros foram adicionados por ano (em azul). Neste gráfico fica claro o crescimento sustentável da comunidade desde a sua primeira edição. 


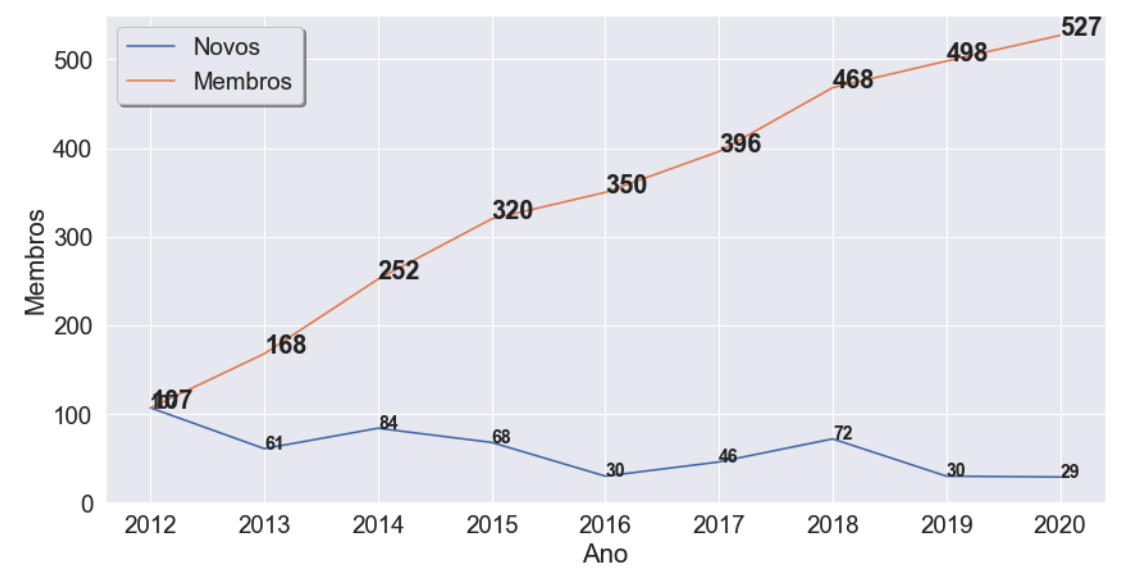

Figura 3. Quantidade de novos membros por edição do BraSNAM.

Em relação a participação nos eventos, $422(80 \%)$ autores participaram de apenas uma edição do evento, 62 (11,76\%) estiveram em duas edições e 43 membros da comunidade participaram de 3 ou mais eventos. Nenhum membro participou de 8 ou mais edições até o momento. Importante notar que a participação considerada é como autor de artigo, uma vez que não consideramos a inscrição no evento e eventual participação como ouvinte. A última análise da comunidade foi em relação a co-autoria. Ao todo, a comunidade BraSNAM conta com 69 componentes conectados e possuem 1.260 pares de autores. Estes resultados estão em consonância com o observado para as instituições, conforme será visto na próxima subseção.

\subsection{Instituições}

Por se tratar de um evento técnico-científico, os membros da comunidade encontramse filiados a uma Instituição. Analisamos a ocorrência das instituições considerando a filiação informada por artigo. Para tal, realizamos um pré-processamento buscando a padronização dos nomes. Isso foi feito transformando os nomes para caixa-baixa, removendo acentuação e depois realizando uma inspeção manual. Por exemplo, universidade de brazilia, university of brasilia e unb foram considerados Universidade de Brasília. No caso de artigos onde, por exemplo, três autores estavam filiados a uma única instituição, computou-se apenas um artigo para ela. Ao todo, 95 instituições se fizeram presentes no BraSNAM até o momento. Na Tabela 1 apresentamos as instituições com mais de 10 trabalhos publicados no evento.

\begin{tabular}{c|c}
\hline Instituição & Quantidade de trabalhos \\
\hline Universidade Federal do Rio de Janeiro & 47 \\
Universidade de São Paulo & 25 \\
Universidade Federal de Minas Gerais & 37 \\
SENAI CIMATEC & 13 \\
CEFET-RJ & 13 \\
Universidade Federal do ABC & 11 \\
Universidade Federal da Paraíba & 11 \\
Universidade de Brasília & 11 \\
Universidade Federal da Bahia & 11 \\
\hline
\end{tabular}

Tabela 1. Instituições com mais de 10 trabalhos publicados no evento.

Em relação a distribuição geográfica, apesar de ser um evento nacional, alguns trabalhos possuíram colaboradores estrangeiros. Sete países fizeram presentes, a saber: 
Estados Unidos (5 artigos), França (3), Argentina (3), Uruguai(2) e Taiwan, Alemanha e Portugal com um trabalho cada. Das 27 Unidades Federativas do Brasil, 9 ainda não se fizeram presentes no BraSNAM como autores. Esta informação pode ser útil para o comitê organizador, a fim de ampliar a divulgação do evento nestes estados. Esta informação pode ser melhor visualizada na Figura 4, onde destacam-se as presenças de Rio de Janeiro, Minas Gerais, São Paulo e Bahia. Observa-se também a participação de estados da região Sul, Norte e Nordeste.

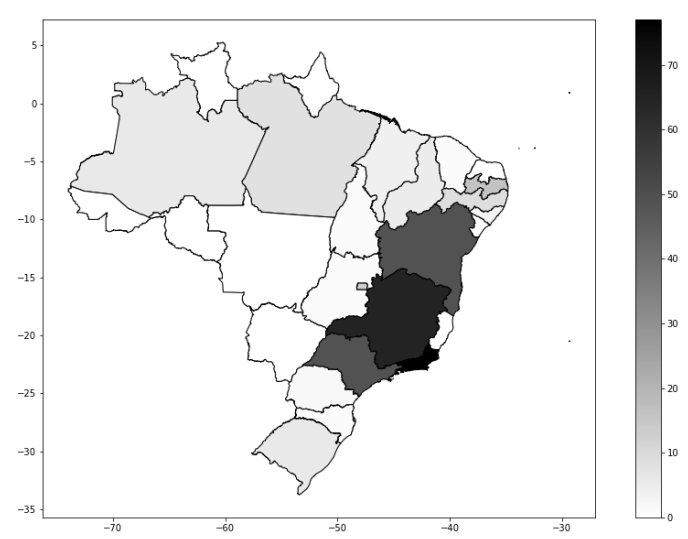

Figura 4. Mapa de calor representando a quantidade de trabalhos por Unidade Federativa.

Mapeamos também as principais relações interinstitucionais que culminaram em uma publicação no BraSNAM. Das 95 instituições, 16 não possuíam nenhuma colaboração interinstitucional e 79 estavam em co-autoria. Conforme mencionado, utilizou-se o software Cytoscape para visualização destes dados. Utilizamos as siglas das instituições para facilitar a visualização. O resultado pode ser visto na Figura 5 ,

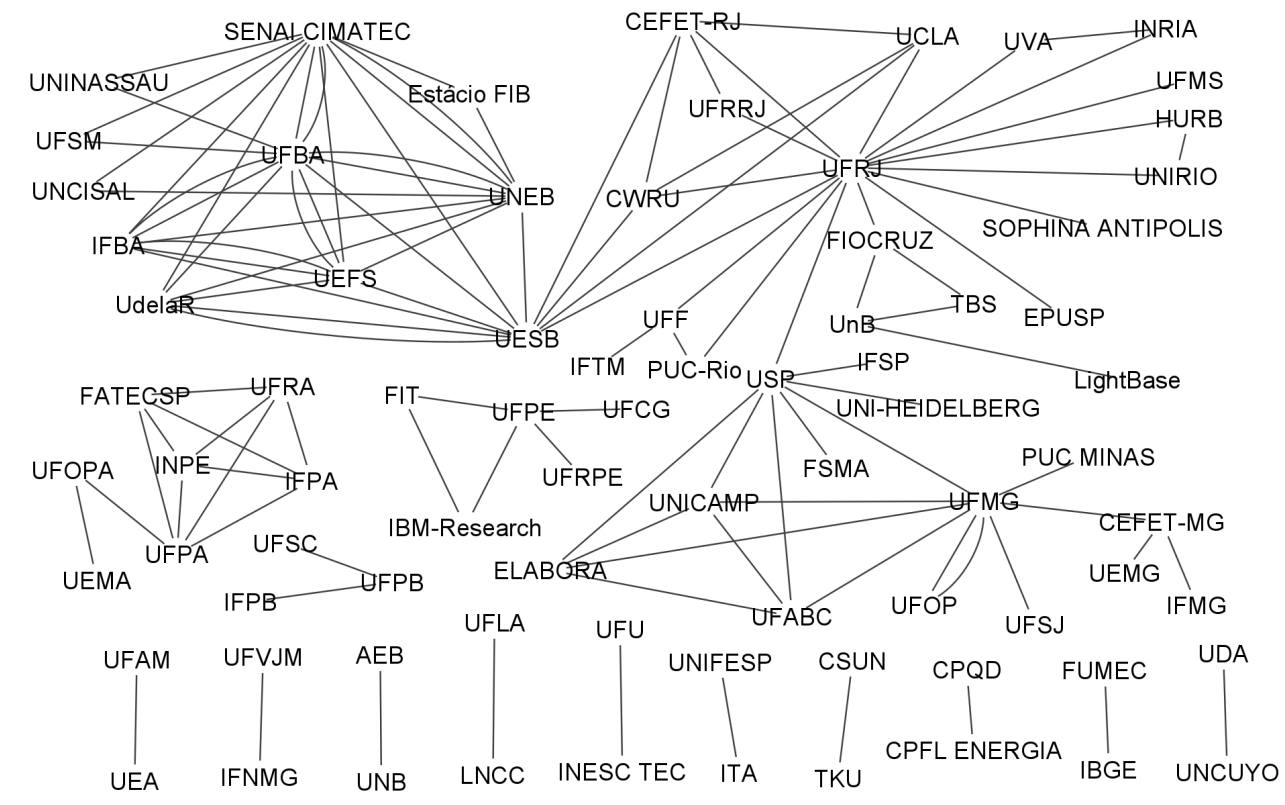

Figura 5. Grafo com as colaborações interinstitucionais mapeadas. 
Por meio da Figura 5 é possível perceber 14 componentes conectados. Interessante notar que o componente maior conta com 44 nós, tendo como Hubs principais as seguintes instituições: Universidade Federal de Minas Gerais, Universidade de São Paulo, Universidade Federal do Rio de Janeiro, e Universidade Estadual do Sudoeste da Bahia. Outras análises da rede foram suprimidas, mas podem ser realizadas com os dados disponibilizados.

\subsection{Impactos do trabalho}

Uma medida importante para avaliar a evolução da comunidade e os assuntos discutidos no evento é a citação dos trabalhos. Conforme apresentado na Seção anterior, verificou-se o número de citações de cada trabalho no Google Acadêmico. Tal informação auxilia na resposta de ambas as perguntas de pesquisa. Até a última consulta, realizada em 03 de maio de 2021, os trabalhos publicados no evento somavam 732 citações, com uma média de 3,2 citações por trabalho. Na Figura 6, apresentamos a distribuição das citações.

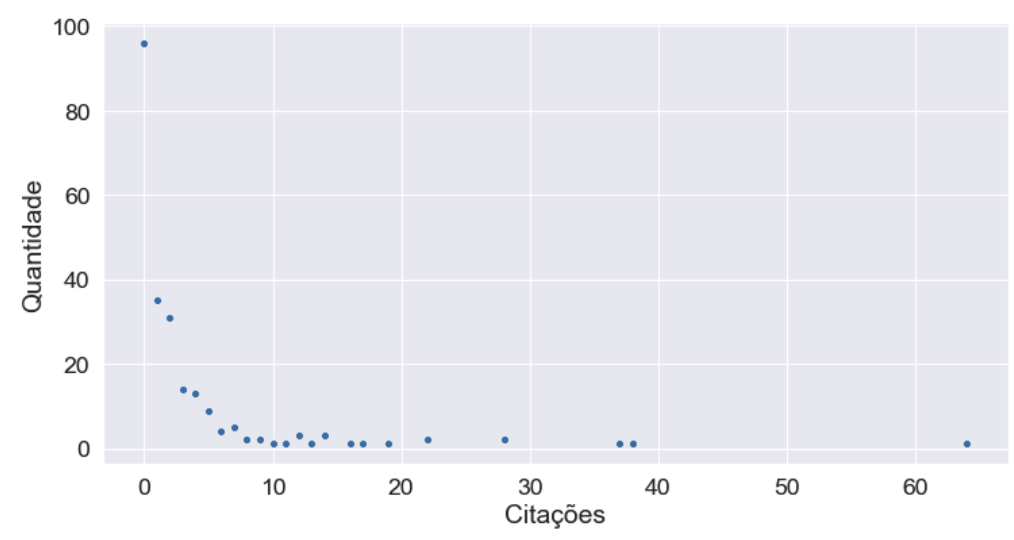

Figura 6. Distribuição do número de citações.

Os dados dispostos na Figura 6 estão em consonância com o esperado, onde a distribuição das citações segue a Lei de Potência. Dos 230 trabalhos publicados, noventa e seis trabalhos, aproximadamente $40 \%$, não possuem nenhuma citação. Conforme aumenta o número de citações, menos trabalhos são observados. Em relação aos temas mais citados, na Tabela 2 apresenta os 10 trabalhos mais citados.

\begin{tabular}{|c|c|c|}
\hline Título do Trabalho & Citações & Ano \\
\hline Minerando e Caracterizando Dados de Currículos Lattes & 64 & 2012 \\
\hline Caracterizando as redes de coautoria de currículos Lattes & 38 & 2012 \\
\hline Análise de sentimento de tweets com foco em notícias & 37 & 2012 \\
\hline $\begin{array}{l}\text { Dinâmica das Relações de Coautoria nos Programas de Pós-Graduação em Computação no } \\
\text { Brasil }\end{array}$ & 28 & 2012 \\
\hline Caracterização de árvores de genealogia acadêmica por meio de métricas em grafos & 28 & 2014 \\
\hline Uma Abordagem Multilíngue para Análise de Sentimentos & 22 & 2015 \\
\hline Offensive Comments in the Brazilian Web: a dataset and baseline results & 22 & 2017 \\
\hline Estudo sobre Pesquisas em Segurança da Informação em Big Data & 19 & 2014 \\
\hline Redes de títulos de artigos científicos variáveis no tempo & 17 & 2013 \\
\hline Modelagem e Caracterização de Redes Científicas: Um Estudo Sobre a Plataforma Lattes & 16 & 2013 \\
\hline
\end{tabular}

Tabela 2. Os 10 trabalhos mais citados do BraSNAM. 
Por meio da análise da Tabela 2 é possível observar que os trabalhos mais citados foram publicados nos três primeiros eventos (2012 a 2014). Apenas dois dos dez trabalhos mais citados foram publicados posterior a isso, 2015 e 2017, respectivamente. Em relação aos assuntos, conforme corroborado por análises a serem apresentadas a seguir, as primeiras edições tiveram uma prevalência de estudos relacionados a redes acadêmicas, seja tratando-se de co-autoria, árvores de genealogia acadêmica ou caracterização de redes científicas. Em um cômputo geral, seis dos dez trabalhos mais citados foram dedicados a esta temática - com três explicitando no título a Plataforma Lattes como fonte de dados. Dois trabalhos foram dedicados a análise de sentimentos e um para segurança da informação.

Por fim, destaca-se que o trabalho mais recente que figura nesta lista envolve um tema com crescente relevância na área, detecção de discurso ofensivo ou de ódio. Além disso, é o único trabalho em língua inglesa desta lista. Nesta senda, observa-se que o evento teve 18 trabalhos publicados em língua inglesa até o momento, sendo sete em 2016, quatro em 2017, um em 2018, dois em 2019 e quatro em 2020. Por ser algo observado apenas nas últimas cinco edições, interpretamos este fenômeno como um sinal de maturidade crescente do evento, que se encaminha para uma maior internacionalização.

\subsection{Temas discutidos}

Para analisar os temas discutidos nos baseamos nas palavras-chaves do artigo. O préprocessamento/padronização foi realizado conforme descrito na Seção2. Ao todo, foram identificadas 469 palavras-chave, com 804 conexões entre elas e 45 componentes conectados. O coeficiente de agrupamento, que mede a transitividade das relações em uma rede, obtido foi de 0,86, considerado elevado, mas esperado dado a diversidade de assuntos discutidos. A interação dos temas discutidos no BraSNAM podem ser visualizados na Figura 7 .

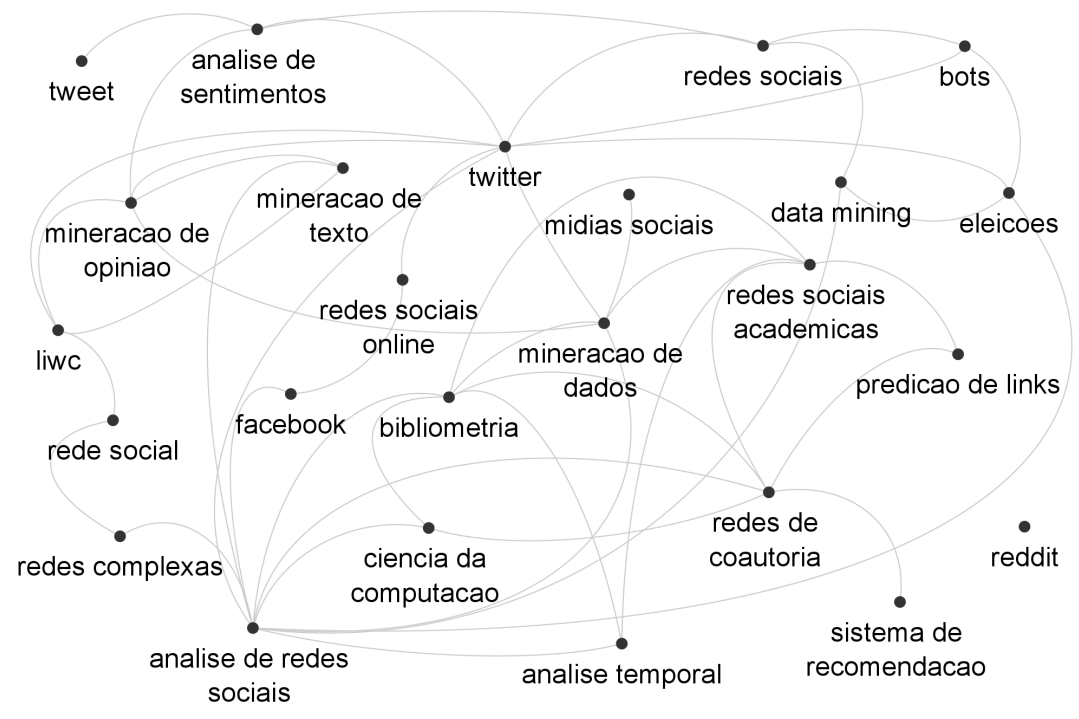

Figura 7. Distribuição do número de citações.

Para fins de legibilidade, aplicou-se um filtro baseado no grau do nó, onde apresentam-se apenas os nós com grau maior que 9 e apenas os componentes conecta- 
dos. Por meio da análise da Figura 7 é possível ver as fontes de dados mais investigadas: twitter, facebook e reddit (como um componente desconectado); aplicações: eleições, redes de coautoria e bibliometria; tarefas: mineração de opinião, análise de sentimentos, detecção de bots, predição de links e análise temporal. Curiosamente, apenas um método aparece neste grafo, o Linguistic Inquiry and Word Count (liwc).

Adicionalmente, realizamos uma filtragem dos nós para os graus entre 7 e 9. A plataforma Lattes passa a aparecer como fonte de dados, com as palavras-chave plataforma lattes e curriculo lattes. Tarefas como modelagem de tópicos e classificação de texto também se fazem presentes. Por fim, destacamos as redes neurais convolucionais, que, apesar de recentemente utilizadas para dados de mídias sociais, têm ganhado destaque crescente.

Como o número de trabalhos publicados não seguia uma distribuição uniforme no decorrer dos anos, a análise temporal foi feita usando as 20 palavras-chave mais frequentes em cada ano. Então, fizemos um sliding, contabilizando por triênio. Conforme esperado o termo Análise de Redes Sociais foi que mais apareceu, em sete edições das nove. Este termo esteve ausente apenas nos anos de 2018 e 2019. O segundo termo mais frequente foi referente a plataforma Twitter. Devido a essa onipresença, estas duas palavras-chave ou termos correlatos (e.g. tweet) foram retirados da linha temporal do BraSNAM. A timeline pode ser visualizada na Figura 8 .

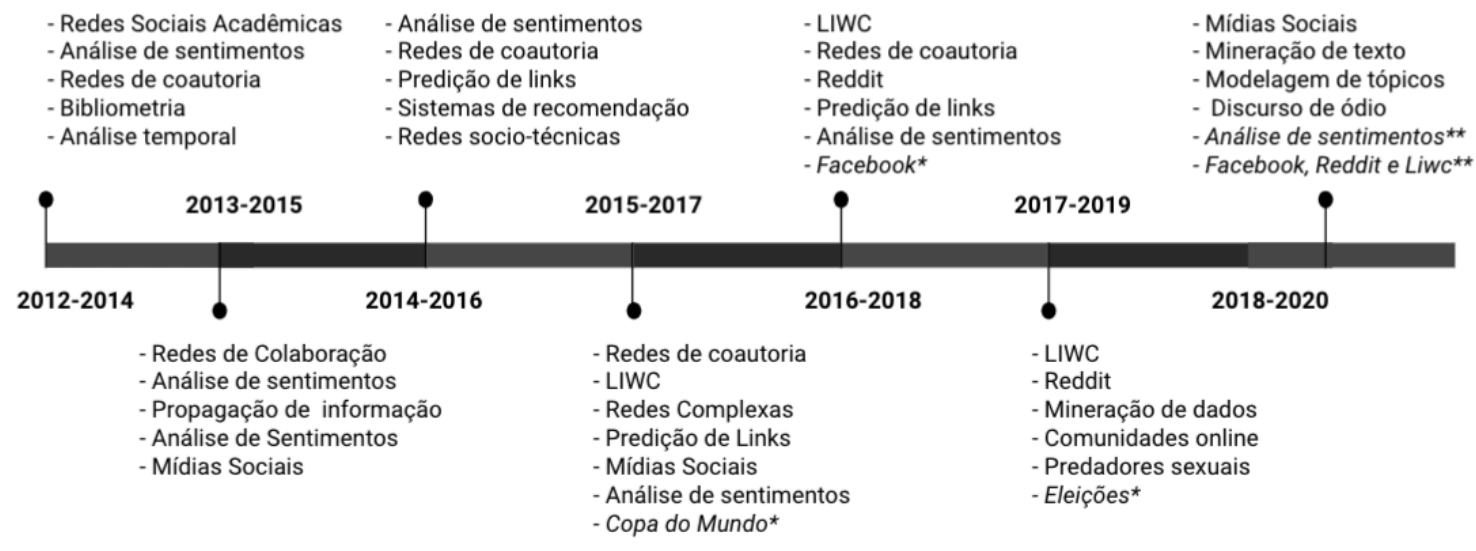

Figura 8. Linha temporal das palavras-chave.

Por meio da análise da Figura 8 é possível perceber que os primeiros anos do BraSNAM tiveram um enfoque em redes sociais acadêmicas, análise bibliométricas e redes de coautoria. Este último termo foi relevante até o triênio 2016-2018. Se faz interessante notar que a partir do quarto marco temporal (2015-2017), o evento passa a apresentar uma diversidade maior de temas, onde destacamos a ocorrência de domínios de aplicação como Copa do Mundo e Eleições (2017-2019) e o Facebook (2016-2018) como fonte de dados - tais termos foram marcados com $*$. Interessante também a popularidade do método LIWC e da plataforma Reddit nas pesquisas discutidas no BraSNAM.

O último marco temporal evidencia ainda mais esta diversidade, com vários termos empatados - destacados com ** na Figura 8. No triênio 2018-2020 percebe-se também um aumento da presença de palavras-chave relacionadas a Inteligência Computacional e Aprendizado de Máquina, como mineração de texto e modelagem de tópicos. 
Evidencia-se o retorno de análise de sentimentos e o surgimento de discurso de ódio. Como quantitativamente as primeiras edições tiveram mais trabalhos publicados, a análise geral apresentada na Figura 7 foi dominada pelos termos mais presentes na primeira edição, evidenciando a relevância da abordagem baseada em linha temporal.

\section{Considerações Finais}

O Brazilian Workshop on Social Network Analysis and Mining (BraSNAM) completa a primeira década de existência neste 2021. O presente artigo apresentou uma análise da trajetória do evento até então, evidenciando o crescimento da comunidade. Artefatos como a rede de colaborações interinstitucionais, a distribuição da participação das Unidades Federativas, as citações dos trabalhos e a escala temporal dos temas discutidos, têm o potencial de guiar o comitê organizador no planejamento estratégico para a expansão sustentável do BraSNAM. Como ações possíveis, destaca-se a ampliação dos esforços de divulgação nos estados com pouca ou nenhuma participação no evento; e disponibilização de uma lista com os trabalhos publicados no último triênio para que os revisores possam estar melhor instrumentalizados na sugestão de referências do evento.

Apesar de todas as análises terem sido feitas considerando um dos autores como juiz, muitas atividades tiveram que ser realizadas manualmente, representando uma ameaça a validade da pesquisa. Visando mitigá-la, os dados e scripts serão disponibilizados em repositório no GitHub para avaliação da comunidade. Outra observação que se faz necessária é quanto a presença de palavras-chave em inglês e português, o que pode gerar ruídos. Como trabalhos futuros, pretende-se levantar os trabalhos convidados para edições especiais de periódicos a partir do evento, avaliando o impacto desta ação. Além disso, a tradução das palavras-chave para o inglês e padronização baseada em similaridade semântica tem o potencial de melhorar a visualização da informação.

\section{Agradecimentos}

Este trabalho foi parcialmente financiado pelo Conselho Nacional de Desenvolvimento Científico e Tecnológico (CNPq) - DT-308334/2020-5.

\section{Referências}

[Barabási et al. 2002] Barabási, A., Jeong, H., Néda, Z., Ravasz, E., Schubert, A., and Vicsek, T. (2002). Evolution of the social network of scientific collaborations. Physica A: Statistical Mechanics and its Applications, 311(3):590-614.

[Chu et al. 2020] Chu, J., Wang, Y., Liu, X., and Liu, Y. (2020). Social network community analysis based large-scale group decision making approach with incomplete fuzzy preference relations. Information Fusion, 60:98-120.

[Dakiche et al. 2019] Dakiche, N., Benbouzid-Si Tayeb, F., Slimani, Y., and Benatchba, K. (2019). Tracking community evolution in social networks: A survey. Information Processing \& Management, 56(3):1084-1102.

[Digiampietri et al. 2017] Digiampietri, L. A., Mugnaini, R., Pérez-Alcázar, J. d. J., MenaChalco, J. P., Delgado, K., and Tuesta, E. F. (2017). Análise da evolucão, impacto e formação de redes nos cinco anos do brasnam. In Proceedings of Brazilian Workshop on Social Network Analysis and Mining (BraSNAM). 
[Kumar et al. 2010] Kumar, R., Novak, J., and Tomkins, A. (2010). Structure and Evolution of Online Social Networks, pages 337-357. Springer New York, New York, NY.

[Lobato et al. 2020] Lobato, F. M. F., Silva Junior, J. L. F., Jacob, A., and Lisboa Cardoso, D. (2020). Social crm: A literature review based on keywords network analysis. In Abramowicz, W. and Klein, G., editors, Business Information Systems Workshops, pages 237-249, Cham. Springer International Publishing.

[Radhakrishnan et al. 2017] Radhakrishnan, S., Erbis, S., Isaacs, J. A., and Kamarthi, S. (2017). Novel keyword co-occurrence network-based methods to foster systematic reviews of scientific literature. PLOS ONE, 12(3):1-16.

[Souza 2015] Souza, I. (2015). Altmetria ou métricas alternativas: conceitos e principais características. AtoZ: novas práticas em informação e conhecimento, 4(2):58-60.

[Su and Lee 2010] Su, H.-N. and Lee, P.-C. (2010). Mapping knowledge structure by keyword co-occurrence: a first look at journal papers in technology foresight. Scientometrics, 85(1):65-79.

[Xu et al. 2020] Xu, Z., Rui, X., He, J., Wang, Z., and Hadzibeganovic, T. (2020). Superspreaders and superblockers based community evolution tracking in dynamic social networks. Knowledge-Based Systems, 192:105377.

[Zhu and Guan 2013] Zhu, W. and Guan, J. (2013). A bibliometric study of service innovation research: Based on complex network analysis. Scientometrics, 94(3):1195-1216.

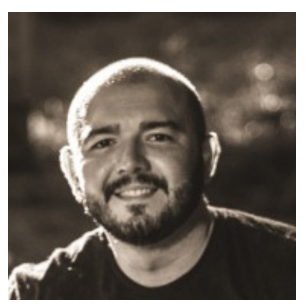

Fábio Lobato é professor no Instituto de Engenharia e Geociências da Universidade Federal do Oeste do Pará (UFOPA) e docente Permanente no Programa de Pós-Graduação em Engenharia da Computação e Sistemas (PECS) na Universidade Estadual do Maranhão (UEMA). É Bolsista de Produtividade Desenvolvimento Tecnológico e Extensão Inovadora do CNPq - Nível 2 (DT-2), atuando nos seguintes temas: Inteligência Computacional, Análise de Redes Sociais e Gestão de Relacionamento com Clientes.

Antonio Jacob Jr Professor assistente do Departamento de

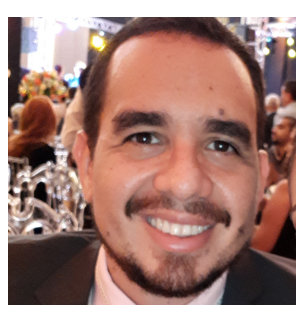
Engenharia da Computação e Docente Permanente no Programa de Pós-Graduação em Engenharia da Computação e Sistemas (PECS) na Universidade Estadual do Maranhão (UEMA). Graduado em Ciência da Computação pela Universidade da Amazônia (2005) e mestre em Ciências da Computação pela Universidade Federal de Pernambuco (2008).

Gleyce Sousa possui graduação em Sistemas de Informação

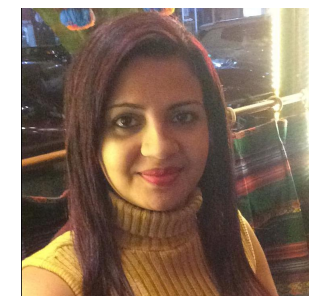
pela Universidade Federal do Oeste do Pará - UFOPA (2020). Atualmente cursa o Mestrado Profissional em Engenharia da Computação e Sistemas na Universidade Estadual do Maranhão (UEMA) e integra o Grupo de Estudo e Pesquisa do Laboratório de Computação Aplicada (LACA) desenvolvendo projetos de gestão de processos para implantação de CRM Social em micro e pequenas empresas. 